\title{
HEADERS AND PAYLOADS INSIDE OF NERVOUS SYSTEM AS LIKE AS DIGITAL COMMUNICATION PROTOCOLS?
}

\author{
O. Nolasco-Jáuregui and J. Leyva-Montiel ${ }^{1}$ \\ ${ }^{1}$ Department of Electronic Design, CINVESTAV, Zapopan, Jalisco, México \\ ${ }^{2}$ Department of Electric Engineering, CINVESTAV, Zacatenco, CDMX, México
}

\begin{abstract}
The Nervous System (NS) transmits information as Structured Messages (SM). The SM is composed by a header followed of a payload. The header indicates to receptor the address where the information needs to be decoded. To validate this proposal this research used the rat NS like a model.
\end{abstract}

\section{KEYWORDS}

Action Potentials, Header, Payload, T-Distributed EM, Message Structure, Rat, Afferent Signal, Inter Spike Interval \& Digital Communication Protocol

\section{INTRODUCTION}

The Nervous System (NS) is a communication network that allows the organism to interact appropriately with this environment [1], [2] y [3]. The human body has sensory component are able to transduce stimuli from the external environment into Action Potentials (APs); the APs will be administered in Processing Centers (PCs) (spinal cord, brain stem, thalamus and cerebral cortex) an then converted to sensations [4]. The PCs command the APs to the motor neurons that will convert into movements of the human body [5].

The study of the neurophysiological mechanisms of sensitivity began in 1925, when Edgar D. Adrian and Yngve Zotterman were the first to record an AP on a sensory nerve [6]. These authors discovered that the nerve carries information from the sensor receiver by modulating the frequency of electrical impulses [7]. Sensory information in the Central Nervous System (CNS) is Processing $(\mathrm{P})$ in stages in the sequential relay nuclei of the spinal cord, brain stem, thalamus and cerebral cortex, known as: Somatosensory Pathways [8]. NS works hierarchically; the sensory receptors transmit information to the first order neurons, these neurons have peripheral axon that forms or innervates somatosensory receptors and a central process that synapses with $2^{\text {nd }}$ afferent neurons in spinal cord or brain stem nucleus; and then to synapses with higher order like thalamic-cortical area [9].

The NS is a complex communications network that interconnects around 100 billion neurons [10]. NS is comparable to any digital communication network because it activates and deactivates systems, guides and coordinates CNS P for afferent and efferent ways [11].

In all digital communication systems, the transmitters and receivers communicate with structured messages. The Structure of Message (SM) is a standard between them [12]. The SM allows the systems to decode and encode information avoiding communication errors [13]. The simplest digital SM would be the header and the payload. In a one-way communication, the header

DOI: 10.5121/ijbes.2017.4103 
indicates the name of the receiver which must decode the information and the payload is the information [14].

\section{HYPOTHESIS}

This research pretends to find the SM of the communication between the CNS and Peripheral Nervous System (PNS). The PNS is the best place to acquire and analyse the neural signal; the reason is because here is the raw signal without $\mathrm{P}$. According to the technical literature, the first $\mathrm{P}$ of the neural signals is in the spinal cord. As the neural signal travels to the upper areas of the brain some information is added until it reaches its final decoding (possibly some of Broadmann's areas). For this reason, this research acquires and works with raw signals from the PNS without P.

The advantage of avoiding the first coding and those of the upper zones is because throughout life each individual has created a series of neural connections at convenience that allow him to survive on his own environment. Another proposal of his research is to assume that all individuals must handle the same type of raw signals. Therefore, like most of us, we have similar anatomies (eyes, nose, mouth, etc.), we shall generate the same SM when we excite a skin sensor, but along various decoding (second and third order neurons) the individual adds "his signature" to his neural signal.

In a pain case, for example, the raw signal must be the same for all individuals, but some people feel more pain than others because each individual has created different neural connections; thus, the decoding will be different for each individual, so each individual feels different perceptions of pain.

In the market there are prostheses that detect the presence of efferent neural signals in the fibers of the nerve and with them move the prostheses. When the prosthesis is moving, the eye feeds back to the brain and the brain learns, from an extend period of time practicing the individual is able to move his prosthesis a convenience with perfect and exact movements, but in this case, the proposal is not to use the natural language of the NS. To have a "natural talking" with the CNS form the PNS the communication must be able to receive an immediate response without a previous learning period.

The main goal of the present research is to decode the "natural talking" between the PNS and CNS. If this research is able to use a natural language between peripheral sensors and CNS, we may be able to find the SM for different stimuli. With the correct SM, we could delete the pain messages in such a way that they changing one or more internal commands; with the correct SM, the NS could be controlled.

\section{METHODS}

The main goal of this research is to find the temporal SM (header and payload) of LowThreshold Mechanoreceptors (LTMRs). To validate this proposal, this research used the rat NS like a model, see Figure 1. The general similarity of mammals NS allows rodent to be used as models, they share equivalences with humans like anatomy, biochemistry, physiology, neurological function and diseases [15], [16] y [17].

The biological protocol of experimentation was submitted on PICUAL (web-platform of CINVESTAV), with the number No. 0133-15 and approbation of CICUAL ${ }^{1}$.

\footnotetext{
${ }^{1}$ Committee Institutional Care and Use of Laboratory Animals of CINVESTAV.
} 
International Journal of Biomedical Engineering and Science (IJBES), Vol. 4, No. 1, January 2017

\subsection{Signal Acquisition}

This research focuses on afferent pathways of the PNS. The mechanical stimuli are in glabrous skin on hind legs of Wistar rats with weights between 200-300g.

\subsubsection{External Stimuli on Skin}

The LTMRs detect the fine touch on the skin. These sensors, when deformed mechanically, produce bursts of APs that are transported through $5-12 \mu \mathrm{m}$ diameter A-beta fibers with conduction velocities of $30-70 \mathrm{~m} / \mathrm{s}$ [18].

The stimuli must be the same along the experimentation. Once the APs burst is detected, the stimuli must be activated in the same area of the patient along the experimentation. The stimuli must be activated only in the LTMRs sensors, so, this proposal use VonFrey filaments calibrated at $4 \mathrm{~g}$-force pressures. With this pressure it is ensuring that the nocireceptors are not activated. Likewise, in order to avoid stimulating thermoreceptors, it is proposed to keep the ambient temperature constant at $20^{\circ} \mathrm{C}$ and rat body temperature at $38^{\circ} \mathrm{C}$ with a thermal gel pad.

\subsubsection{Intraneural Electrodes for Recording}

The signal acquisition electrode is an invasive type [19]. The electrode is placed on the sciatic nerve. The electrode is an array of 32 USEAs, "Utah Slanted Electrode Array"; they are multielectrodes array of microscopic needle types [20]. They acquire intraneural spikes ranging to $15 \mu \mathrm{V}$ amplitude [21].

The recording capabilities may be affected by biocompatibility with the patient [22], [23] y [24], traumatic nerve damage at electrode insertion [25], a bad mechanical adjustment due to the rigid electrode structure [26], tissue softness [27], non-penetration to fascicle [28], and the forces by the immobilization of the transcutaneous connection cables have been subjects treated in many publications in the last years [29], [30] y [31].

\subsubsection{Electronic Circuit of Data Acquisition}

The acquisition system is ${ }^{\circledR P l e x o n}$. The characteristics of neural signal recording were: $40 \mathrm{kSps}$, gain of $20 \mathrm{~K}$, pre-amplification of $1 \mathrm{~K}$, the noise of the circuitry is measured with the cursors manually and once the patient is connected to the reference circuitry and after the patient is within Faraday's cage. The noise of the circuitry was $40 \mu \mathrm{V}$ and a manual threshold of $-7.263 \mu \mathrm{V}, 32$ micro-electrodes around 700 impedance values, the channels recording in real time with recording times are about $60 \mathrm{~s}$. The timing of the stimuli was 5 per recording in the seconds: 6,9 , 13, 17 and 20. The separation time of each stimulus is to ensure the recovery of the NS. Offline Spike Sorter software of ®Plexon offers the T-Distribution EM Scan, Valley Seeking and KMeans spike detection tools, all of them based on PCAs (Principal Components Analysis). Jutta Kretzberg, et. al., evaluated the performance of two algorithms: T-Distributed EM and Valley Seeking spikes (which ${ }^{\circledR}$ Plexon Offline Spike Sorter offers in its list of algorithms). They found that T-Distributed EM is clearly superior to using artificial data and added noise [32]. Hartigan and Wong in 1978, compares the K-Means and T-Distributed EM spike clustering algorithms [33]. The K-Means algorithm is a specific case of T-Distributed EM because it is based on Euclidian distances and $k$ observations; the problem arises when comparisons are made between global distances and several observations. In contrast, the T- Distributed EM is not based on the Euclidean distances it uses a normalized quantities (values ranging from 0 to 1 ) and the maximum likelihood. In this proposal, we select T-Distributed EM for the detection of spikes in the recordings. 


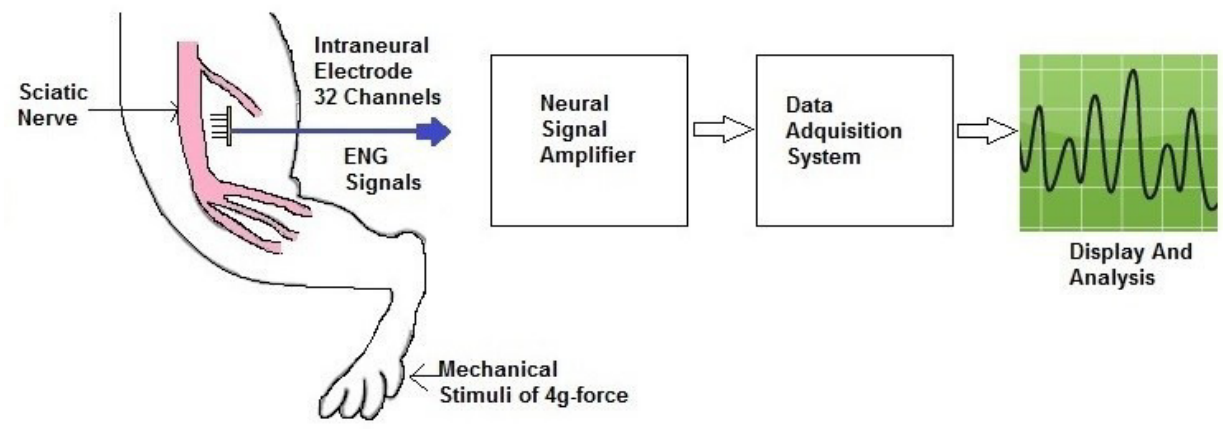

Figure 1. Schema of neural signal acquired from the rat PNS. ENG-Electroneurogram.

\subsection{Signal Analysis}

This research proposes to find the SM of the communication between the PNS and CNS through the insertion of subsets. The basis of the scanning is the matching elements within the symbol space. The space symbol is the recording file converted to the standard language, see Table 1. The space will be regrouped into finite subsets depending on the number of symbolizes created in the space recording time. The regrouping will be changing depending on the matching elements. The largest match subset within the subsets of space will be named the Root Element (RE).

\subsection{ISDMessage-Symbols Software Tool}

The ISDMessage-Symbols (Inter Spikes Distances to Symbols) tool is a proposal of this document that converts a PLX file to standard language, sees Table 1, forms subsets, and scans the recording and searches for the intersection of the elements into the subsets.

The intersected subsets could be regrouped depending on the number of intersecting internal elements:

- First Level: There are those subsets that intersect in 3 or 2 elements. That is, 3 or 2 of its elements are equal to the RE.

- Second Level: There are those subsets that intersect in 1 of its elements. That is, 1 of its elements is equal to the RE.

- Third Level: Are those remaining subsets. Generally, in this level these subsets intersect its elements with subsets of first and second level.

\subsection{Symbols}

The standard language consists of 30 symbols representing the spike-spike distance in milliseconds in neural recordings.

Table 1. Standard Language

\begin{tabular}{|l|l|l|l|}
\hline \multicolumn{4}{|l|}{ Symbol $<$ ó $=$ time $(\mathbf{m s})$} \\
\hline $1<=1.772$ & $2<=3.375$ & $3<=5.316$ & $4<=7.088$ \\
\hline $5<=10.631$ & $6<=14.175$ & $7<=17.719$ & $8<=24.856$ \\
\hline $9<=31.993$ & $10<=35.129$ & $11<=38.235$ & $12<=45.875$ \\
\hline $13<=53.406$ & $14<=56.542$ & $15<=64.112$ & $16<=85.524$ \\
\hline $17<=128.348$ & $18<=153.329$ & $19<=263.899$ & $20<=338.899$ \\
\hline $21<=374.571$ & $22<=438.037$ & $23<=517.333$ & $24<=731.454$ \\
\hline $25<=802.827$ & $26<=838.514$ & $27<=874.201$ & $28<=909.887$ \\
\hline $29<=945.574$ & $30<=1.05263 \mathrm{~s}$ & & \\
\hline
\end{tabular}




\subsection{Calibration Process}

The calibration process is a proposal to choose the channels with which to work along the characterization. For each in-vivo experiment its necessary recording 2 files (previously analysed by the algorithm of T-Distributed EM):

- Baseline.plx

- Lidocaine.plx

The Baseline.plx file has the recording of the biological noise (the signal acquired from the system without stimulation). The Lidocaine.plx file is recording once placing the drug lidocaine over the nerve; it does immediately inhibit neural activity. Then, a result of comparing the same channels of both files is a channel with neural activity. A neural channel has a neural activity increased. Finally, a correlation method is used between the neural channels to avoid redundant channels.

\section{RESULTS}

\subsection{Patient\#1 4g-force Subsets}

As a result of the calibration process, from the patient\#1 channel chosen are: 1, 3, 6, 12 and 32. In this case, these channels recorded 5 different fibers, depending on the type of fibers they could be activated conditional to the intensity of the stimuli, or they remain in their inactive state unless there is spontaneous activity.

The recording file $44.5620 \mathrm{k} 01$.plx has a cutaneous stimulation with the VonFrey filament number 4.56 equivalent to $4 \mathrm{~g}$-force. In this file the channel 6 increased in activity with respect to the Baseline.plx recording, which means, that it had an A-beta fiber. The recording file f4.562k01.plx has 1018 spikes in 45.9134 seconds.

The Table 2 is the result of the recording f4.5620k01.plx, with 280 different subsets and 300 total subsets. The Table 2 shows 280 subsets, like these ones: 34 9, $355,349,355,366,389,39$ $5,435,438,467,537,545,586,588,595,5816,5819,653,659,663,666,679$, $688,735,779,795,799,849,869,873,889,896,899,939,949,95 \quad 6,968,616$ 5, $6196,6198,6611,6618,67$ 17, 68 16, 69 16, 69 17, 711 8, 712 9, 715 6,7 15 6, 715 8 , and others.

Table 2. Subsets (clusters of three symbols).

\begin{tabular}{|c|c|c|c|c|c|c|c|c|c|}
\hline \multicolumn{2}{|l|}{ Register 1 } \\
\hline 349 & 969 & 1694 & 9517 & 16166 & 968 & 1678 & 16135 & 16813 & 679 \\
\hline 355 & 973 & 1699 & 9518 & 16169 & 6618 & 6611 & 31612 & 16816 & 688 \\
\hline 366 & 984 & 1739 & 31917 & 91613 & 6717 & 9716 & 31619 & 16910 & 735 \\
\hline 389 & 988 & 1749 & 41216 & 91716 & 6816 & 9819 & 171517 & 16911 & 779 \\
\hline 395 & 996 & 1767 & 41617 & 91718 & 6916 & 9913 & 171612 & 17616 & 795 \\
\hline 435 & 999 & 1776 & 41817 & 101214 & 6917 & 10135 & 171616 & 17815 & 799 \\
\hline 438 & 1085 & 1779 & 51218 & 111215 & 7118 & 10179 & 171717 & 17913 & 849 \\
\hline 467 & 1086 & 1838 & 51517 & 111516 & 7129 & 10312 & 16173 & 19157 & 869 \\
\hline 537 & 1088 & 2179 & 61319 & 121019 & 7156 & 10711 & 16176 & 181110 & 873 \\
\hline
\end{tabular}


International Journal of Biomedical Engineering and Science (IJBES), Vol. 4, No. 1, January 2017

\begin{tabular}{|c|c|c|c|c|c|c|c|c|c|}
\hline 545 & 1089 & 3103 & 61517 & 121112 & 7158 & 11126 & 16177 & 181613 & 889 \\
\hline 586 & 1166 & 3138 & 61611 & 121216 & 7163 & 11137 & 16195 & 181617 & 896 \\
\hline 588 & 1188 & 3148 & 61719 & 121812 & 7168 & 11615 & 16416 & 17916 & 899 \\
\hline 595 & 1238 & 3153 & 61816 & 121916 & 7178 & 11619 & 16610 & 18918 & 939 \\
\hline 599 & 1239 & 3169 & 62014 & 131217 & 7714 & 11817 & 16716 & 19128 & 949 \\
\hline 653 & 1245 & 3418 & 71015 & 131315 & 7916 & 11917 & 171312 & 19136 & 956 \\
\hline 659 & 1255 & 3712 & 71213 & 131712 & 7919 & 12158 & 171313 & 191710 & 71617 \\
\hline 663 & 1273 & 3817 & 71517 & 151217 & 8127 & 12173 & 171317 & 17154 & 81310 \\
\hline 666 & 1275 & 4109 & 71616 & 151312 & 8128 & 12174 & 17169 & 91517 & 81315 \\
\hline 12178 & 191810 & 81316 & 161012 & 8616 & 13105 & 17183 & 81513 & 161016 & 8817 \\
\hline 12183 & 91312 & 81516 & 161210 & 8910 & 13138 & 17218 & 81610 & 161215 & 9128 \\
\hline 12188 & 91413 & 81612 & 161417 & 9153 & 13812 & 16917 & 81615 & 161418 & 9154 \\
\hline 81616 & 161613 & 9173 & 14145 & 17118 & 81619 & 161617 & 9178 & 14618 & 17416 \\
\hline 81718 & 161718 & 9316 & 15410 & 17418 & 81916 & 171014 & 9413 & 15813 & 17517 \\
\hline 4159 & 4174 & 4314 & 5116 & 5139 & 5167 & 5517 & 5816 & 5819 & 6165 \\
\hline 6198 & 6319 & 8199 & 8419 & 8518 & 17196 & 31216 & 17105 & 171117 & 171213 \\
\hline 1287 & 1299 & 1337 & 1358 & 1359 & 1384 & 1393 & 1397 & 1456 & 1469 \\
\hline 1497 & 1549 & 1643 & 1653 & 1663 & 151712 & 151719 & 151815 & 13126 & 13516 \\
\hline 13818 & 91014 & 91019 & 6196 & 171815 & 9414 & 15814 & 9516 & 16125 & 19169 \\
\hline
\end{tabular}

The Table 3 shows in red the first level subsets, the second level in blue and third level in green. The subsets are clusters of three symbols. The RE of this space symbols (with the highest occurrence) is 395, which means, the cluster of symbols 3, 9 and 5. The symbols, 1 and 2, were replaced by the letter " $x$ " because they were very small distances. The rest of symbols that were not subset elements appear in black.

The Table 3 show only 28 de 79 total messages found on f4.5620k01.plx file; the starts message is defined with the "<<" character ant the message ends with " $>>$ ". See Figure 2, register $=1$.

Table 3. Message-Symbols

\begin{tabular}{|l|}
\hline Channel 6 \\
\hline $308 \times 7129111215161016>>>$ \\
$8910<<<1019 \times 12174191710>>>$ \\
$939<<<1719681316>>>$
\end{tabular}




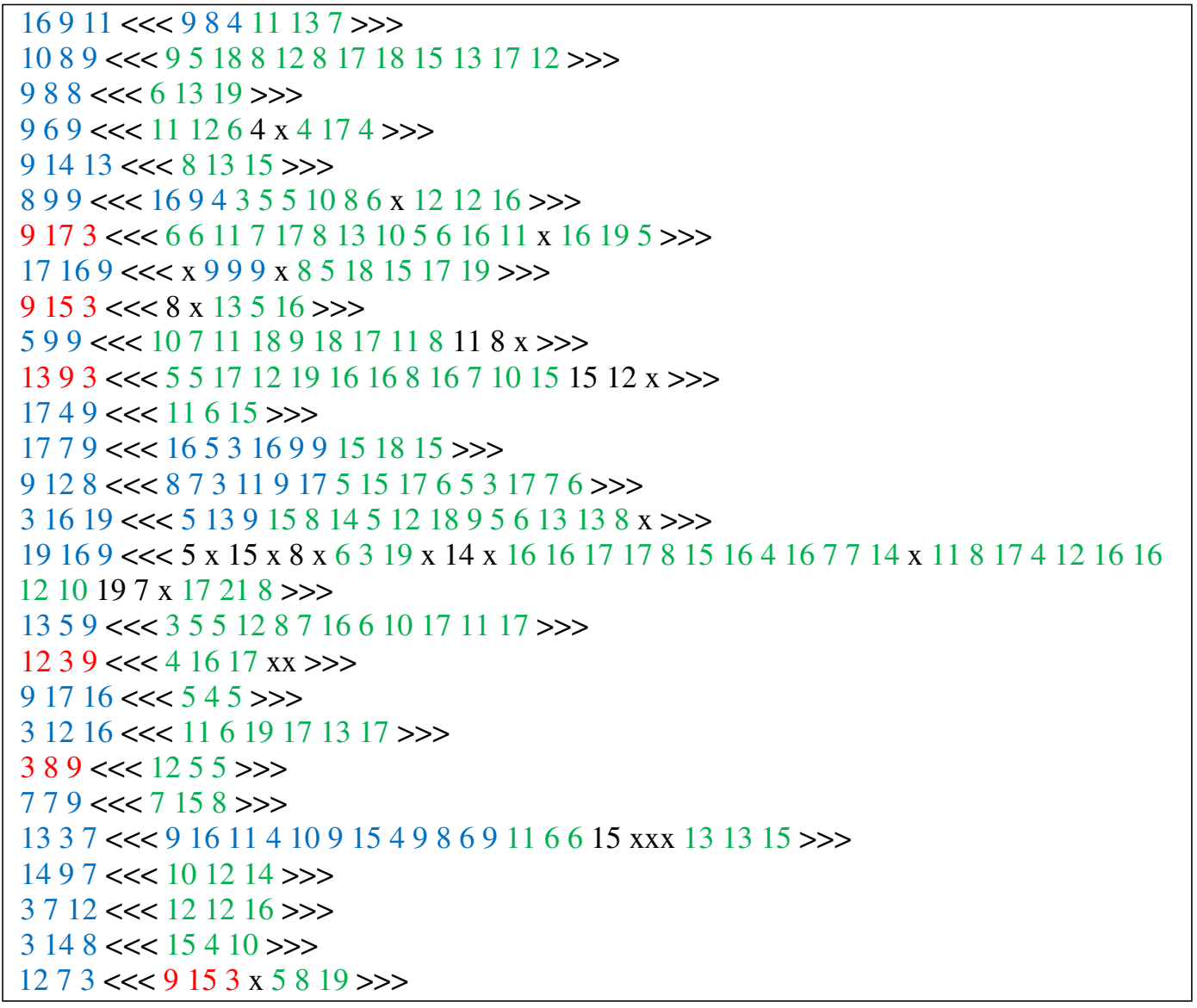

\subsection{Patient\#1 4g-force Messages}

The ISDMessage-Symbols scanning tool allows the identification of patterns. The matching pattern on all recordings is:

- High priority subsets are followed by low priority subsets and in this sequence they reappear throughout the recording.

The high priority subsets are a proposal of this document that identifies the subsets of greater occurrence throughout the recording and that share symbols with the RE, as was the case of subsets of levels 1 and 2 .

The subsets of low priority are the subsets that intersect each other with matching elements but with fewer occurrences within the recording and that do not share elements with RE, as is the case of subsets of level 3.

Finally, the SM is defines like:

- The start Message is a subset of high priority and the end of Message is a subset of low priority.

\subsection{Patient\#1 4g-force Headers}

The Table 3 shows 28 of 49 total headers found on f4.5620k01.plx file, like: $389,395,435$, $537,599,679,735,779,869,889,899,939,939,949,968,969,973,988,10$ $89,1238,1239,1273,1299,1337,1359,1393,1497,1663,1749,1779,1883,217$ 9, $3169,3712,3817,4159,4314,6916,712$ 9, $7919,8910,9128,9153,9173,93$ 
16 y 94 13. See Figure 3, register $=1$.

\subsection{Patient\#1 4g-force Payloads}

The Table 3 shows 20 longitudes payloads, these have since: 3 symbols, 5, 6, 3 symbols, 5, 6, 7, $8,9,10,11,12,13,15,16,18,19,20,21,22,38,40,43$, with an occurrence respectively of 18 , $3,10,3,5,10,4,4,7,1,2,2,2,1,1,1,2,1,1$ y 1 . See Figure 4, register $=1$.

\subsection{Others Recording in Patient\#1 4g-force}

On channel 6, the f4.5620k02.plx register had 856 spikes in $36.7273 \mathrm{~s}$ of recording, the ISDMessage-symbols software tool found 233 different subsets with 239 total subsets, see Figure 2 , register $=2$.

The RE is 7517, which means, it is a cluster of 7, 5, and 17 symbols. The Figure 3, register $=2$, shows its headers values. The recording has 32 Messages with payloads longitudes of 3, 6, 8, 9, $10,12,13,15,17,18,24,25,35,36,37,39,42,54,59,77$, with a respectively occurrence of 4 , $1,1,3,1,1,2,3,1,2,2,1,2,1,1,1,1,1,1$ y 2 . See Figure 4, register $=2$.

On the channel 6, the $44.5620 \mathrm{k} 03$.plx register had 1331 spikes in $56.385 \mathrm{~s}$ of recording; the ISDMessage-symbols software tool found 353 different subsets with 369 total subsets.

The 659 is the RE, that means, it is a cluster of 6, 5 and 9 symbols, see Figure 2, register $=3$. The Figure 3, register $=3$, shows its headers values. This register has 94 messages, with longitudes in their payloads since 3 symbols, 4, 6, 7, 8, 9, 10, 11, 12, 13, 14, 15, 16, 17, 18, 20, 21, 23, 25, 30, 38 with their respectively occurrence of 14 times, $1,12,3,5,8,6,5,6,7,2,5,1$,

$3,5,4,2,1,2,1$ y 1 . See Figure 4, register $=3$.
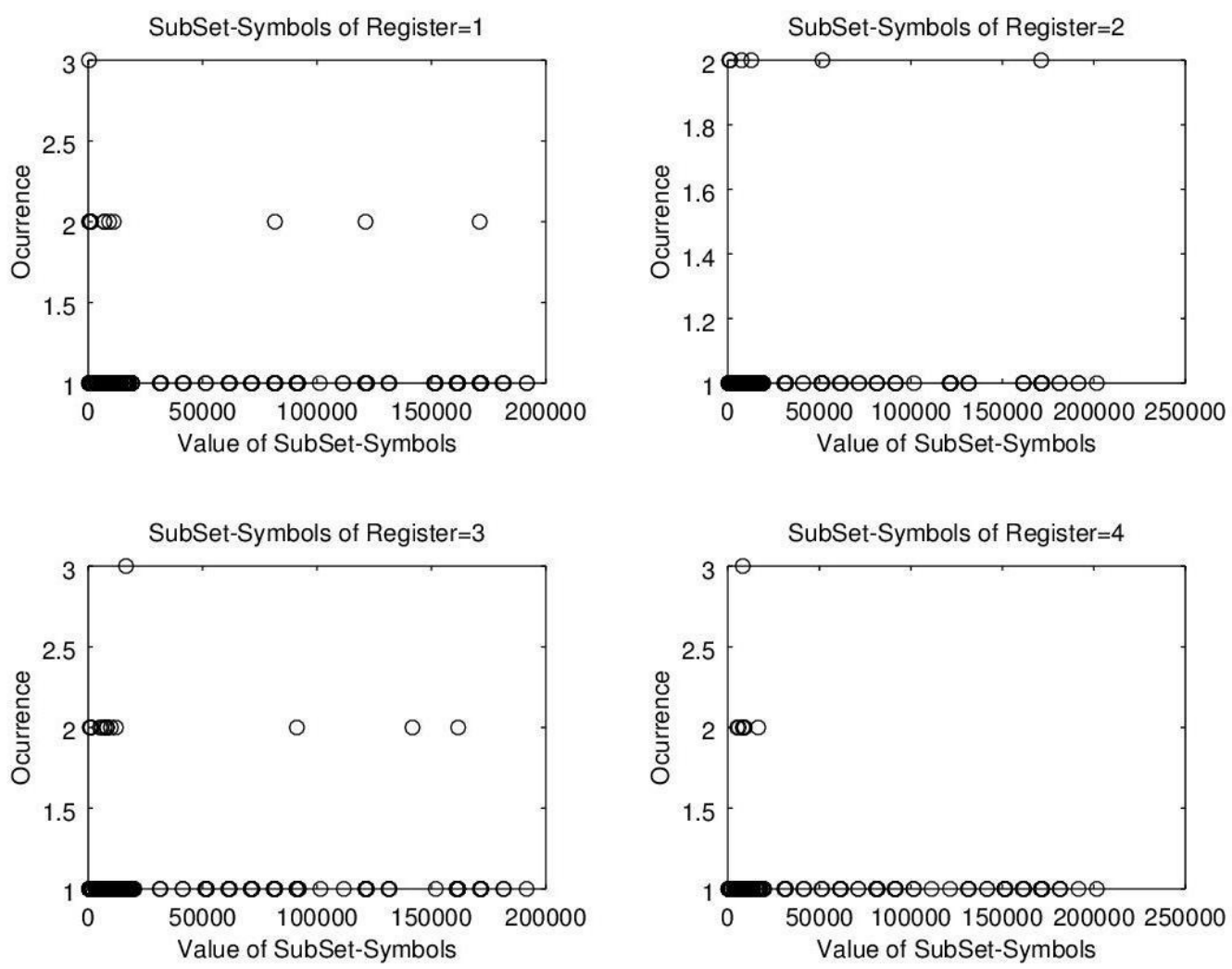

Figure 2. Subset comparative result of ISDMessage-Symbol tool from Patient\#1 with 4g-force. 
International Journal of Biomedical Engineering and Science (IJBES), Vol. 4, No. 1, January 2017
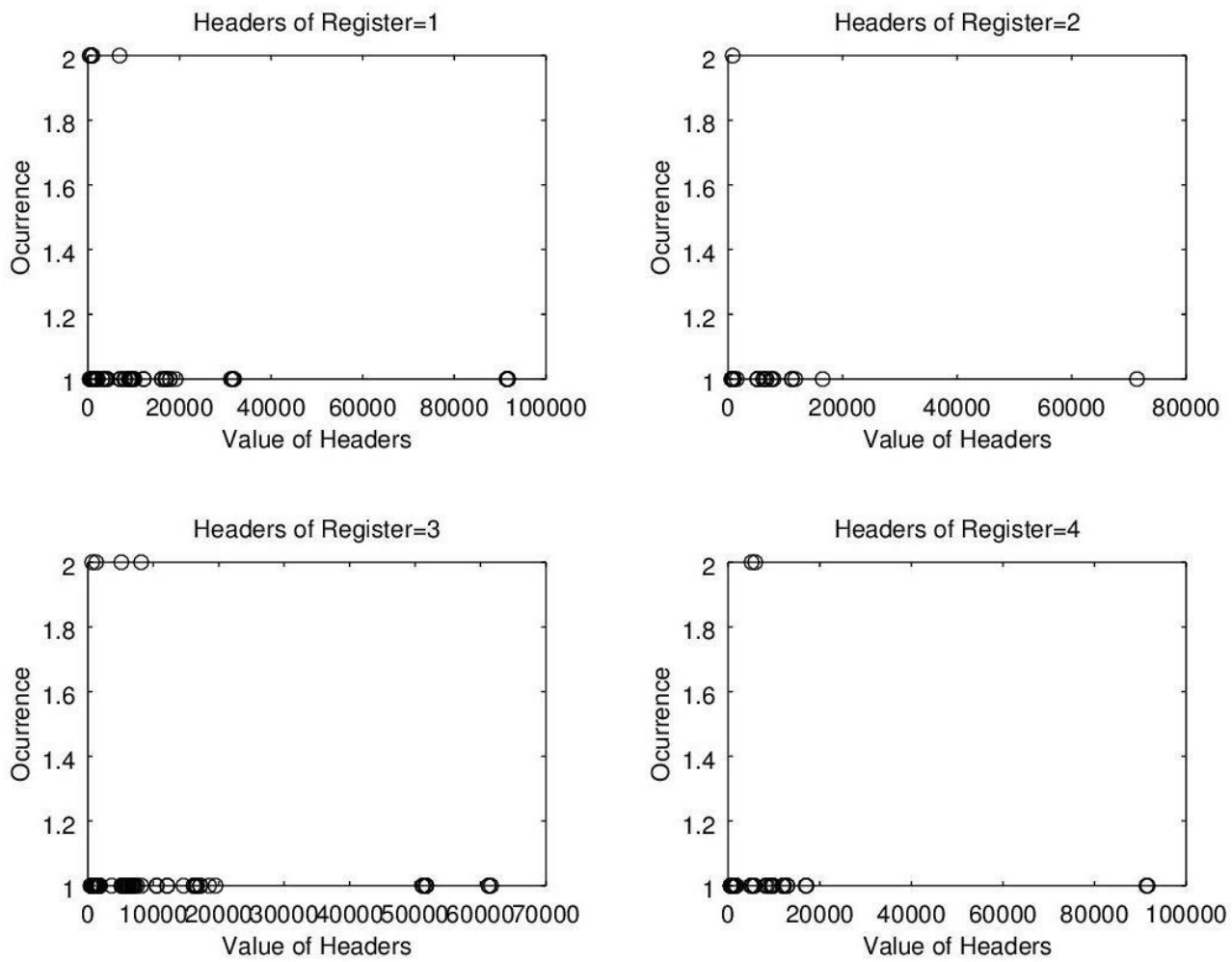

Figure 3. Header comparative result of ISDMessage-Symbol tool scanning from Patient\#1 with 4g-force.
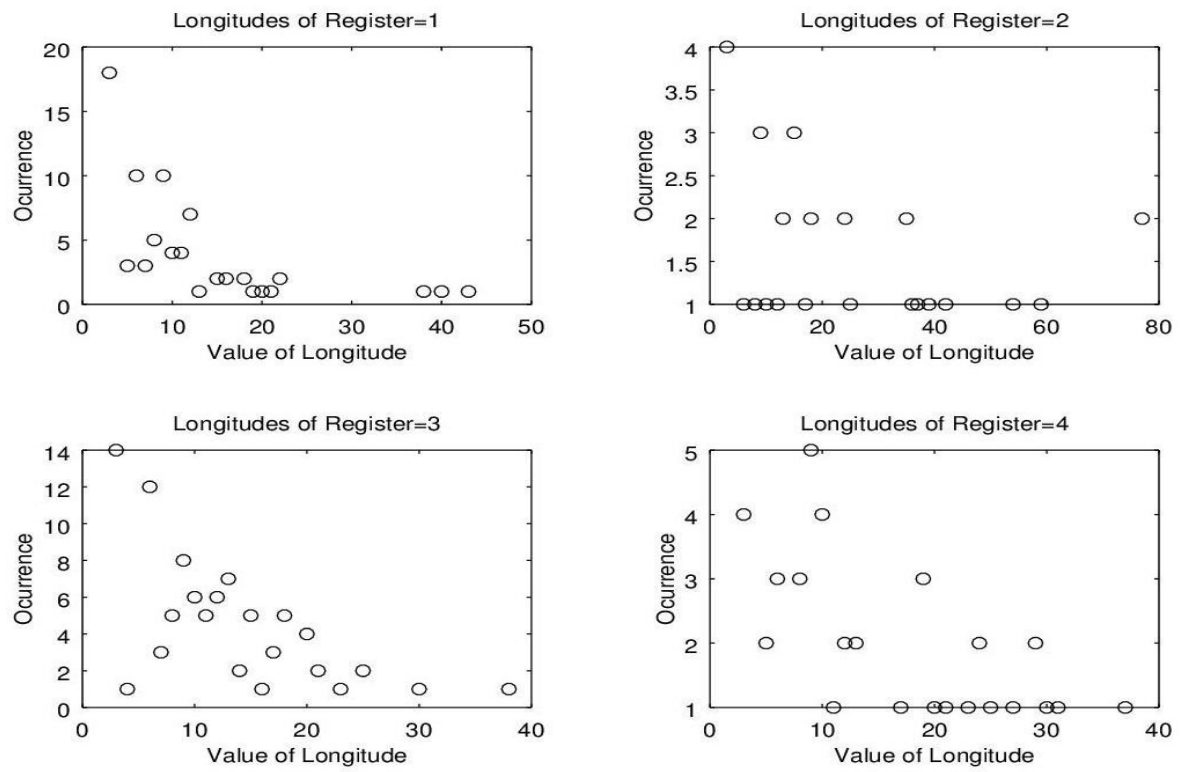

Figure 4. Longitude comparative result of ISDMessage-Symbol tool scanning from 4 different recordings of Patient\#1 with $4 \mathrm{~g}$-force.

On the channel 6, the $44.5620 \mathrm{k} 04$.plx register has 727 spikes in $32.0499 \mathrm{~s}$ of recording; the ISDMessage-symbols software tool found 199 different subsets with 207 total subsets.The RE is 5916, therefore, it is a cluster of 5, 9 and 16 symbols, see Figure 2, register $=4$. The Figure 3, 
register $=4$, shows its header values. This register has 42 , Messages, with longitudes in their payloads since 3 symbols, 5, 6, 8, 9, 10,11, 12, 13, 17, 19, 20, 21, 23, 24, 25, 27, 30, 31 у 37, with their respectively occurrence of 4 times, $2,3,3,5,4,1,2,2,1,3,1,1,1,2,1,1,2,1,1$ y 1. See Figure 4, register $=4$.

\section{CONCLUSIONS AND FUTURE WORK}

The SNP is the best place to acquire the signal without P, lacking of the "signature" of each person. The Figure 2, Figure 3, Figure 4 are the results from recordings in the same patient under the same experimental situations it is easy to notice the similarity between the registers.

The repetitiveness of the occurrences on the subsets tell us about an intelligent structures defined within the bursts of APs by cutaneous stimulating the skin of the patient. The repeated sequence of high priority, low priority and high priority subsets allows us to define the start and end of the message.

Initially, this research does not pretend to make a comparative among patients because it considers the individual as a unique being that by itself anatomy could handle raw signals different from than other patients.

Due to the uniqueness of the system, patients are managed independently to such an extent that prior to the initiation of the analysis the calibration process is performed and possibly in a future, the message structures shall be calibrated to each patient before being used.

The matching structures found in patient\#1 (as shown in the results section) became a breakthrough for this research. The coincidences in the structures inform us that the NS handles the stimulus by intelligent communication with temporized structure. And the NS must be handling a standard language known by the internal systems. Decoding the cutaneous communication code is practically the domain of the afferent system of PNS.

The possible reason why this research did not obtain a defined structure but only coincidences on subsets and not a complete matching may be the consideration of the "simple structure" proposed in the messages.

This document proposed a simple structure, header and payload, of the communication protocol and may not be adequate; and one should think in a complex structure with error detection algorithms, parity checking mechanisms; and protocols compared to telecommunication systems. Indeed, the coincidences in the subsets and their intersections speak about an intelligent and structured communication.

The nerve anatomy itself indicates that the myelin sheath prevents fibers from interfering with their signals with others. Just like wires have an insulation sleeve to prevent their signals from being limited or interfered with. Thousands of fibers travel through the nerve, and phenomena such as saltatory conduction communication cannot be a crude communication that increases or decreases its frequency with the intensity of the stimulus. APs follow a structuring, are interpreted and codified with a standard that can be compared to any digital communication. The goal of this research is to find out what is the communication standard by reverse engineering.

In future work this research pretend use a message with a more complex structure with a calibration process; do not pretend to make a comparative among patients because it considers the individual as a unique.

\section{ACKNOWLEDGEMENTS}

The authors are very grateful with Dr. Mario Romero for his counseling and bioengineering laboratory facilities at the University of Texas at Dallas. This work has supported by the National Council for Science and Technology of México (CONACYT). 
International Journal of Biomedical Engineering and Science (IJBES), Vol. 4, No. 1, January 2017

\section{REFERENCES}

1. Hall, J. E. (2015). Guyton and Hall textbook of medical physiology. Elsevier Health Sciences.

2. Mattew N. Levy, Bruce M. Koeppen, Bruce A. S. (2009). Fisiología, Madrid: Elsevier 6th Ed.

3. Cardinali, Daniel P. (1991). Manual de neurofisiología. Ediciones Díaz de Santos.

4. Fox, Stuart Ira. (2003). Fisiología humana. McGraw-Hill Interamericana de España, 12th Ed.

5. Kandel, Eric R., et al. (2000). Principles of neural science. New York: McGraw-hill, 1st Ed.

6. Zotterman, Y. (1939). Touch, pain and tickling: an electro-physiological investigation on cutaneous sensory nerves. The Journal of physiology, 1939, vol. 95, no 1, p. 1.

7. Roudaut, Yann, et al. (2012). Touch sense: functional organization and molecular determinants of mechanosensitive receptors. Channels, vol. 6, no 4, pp. 234-245.

8. Misgeld, T. N. (2007). In Vivo imaging of signal axons in the mouse spinal cord. Nat. Protoc., Vol. 2, pp. 263-268.

9. Kanamori, A. C. (2010). In Vivo imaging of retinal ganglion cell axons within the nerve fiber layer. Invest. Ophthalmol. Vis. Sci., Vol. 51, pp. 2011-2018.

10. Leung., C. (2011). Long-term in vivo imaging and measurement of dendritic shrinkage of retinal ganglion cell. Invest. Ophthalmol. Vis. Sci., Vol. 52, pp. 1539-1547.

11. Sivagnanam, Subhashini, et al. (2013). Introducing the Neuroscience Gateway. EnIWSG”. 2013.

12. Comer., D. E. (2000). Internetworking with TCP/IP - Principles, Protocols and Architecture (4th ed.). USA.: Prentice Hall.

13. Holzmann., G. J. (1990). Design and Validation of Computer Protocols. USA.: Prentice Hall.

14. Perlman, R. (2000). Interconnections: Bridges, Routers, Switches, and Internetworking Protocols: Bridges and Routers (APC). Boston, MA, USA.: Addison-Wesley Longman Publishing Co., Inc.

15. Treuting, Piper M.; Dintzis, Suzanne M. (2011). Comparative Anatomy and Histology: A Mouse and Human Atlas (Expert Consult). Academic Press, 1st Ed.

16. Petrusz, P. (2005). The Handbook of Experimental Animal The laboratory Rat. University of North Carolina, Chapel Hill, U.S.A.: Edited by Georg Kringe. 2nd Ed.

17. Javier Longás Valién, J. C. (2007). Manual del anaestesiologo URPA y REA. Majadahonda (Madrid): Ergon. C/ Arbolea, 1st Ed.

18. Ma Reina, A. L. (2000). Morfología de los nervios periféricos, de sus cubiertas y su vascularización. Congreso de Anestesiología y Reanimación (pág. 5). Madrid: Valmojado.

19. Wark, H. A. C., et al. (2013). A new high-density (25 electrodes/mm2) penetrating microelectrode array for recording and stimulating sub-millimeter neuroanatomical structures. Journal of neural engineering, vol. 10, no 4, p. 045003.

20. Fujishiro, Akifumi, et al. (2014). Flexible neural electrode arrays with switch-matrix based on a planar silicon process. Electron Device Letters, vol. 35, no 2, pp. 253-255.

21. Rothe, Joerg, et al. (2014). Fully integrated CMOS microsystem for electrochemical measurements on $32 \times 32$ working electrodes at 90 frames per second. Analytical chemistry, vol. 86, no 13, pp. 6425432.

22. Fitzgerald, James J., et al. (2012). A regenerative microchannel neural interface for recording from and stimulating peripheral axons in vivo. Journal of neural engineering, vol. 9, no 1, p. 016010.

23. Lee, Woong Hee, et al. (2013). Microfabricated sampling probes for in vivo monitoring of neurotransmitters. Analytical chemistry, vol. 85, no 8, pp. 3828-3831.

24. Zaaimi, Boubker, et al. (2013). Multi-electrode stimulation in somatosensory cortex increases probability of detection. Journal of neural engineering, vol. 10, no 5, pp. 056013.

25. Pongracz, Anita, et al. (2013). Deep-brain silicon multielectrodes for simultaneous in vivo neural recording and drug delivery. Sensors and Actuators B: Chemical, vol. 189, p. 97-105.

26. Felete, Zoltán, et al. (2015). Experimental study on the mechanical interaction between silicon neural microprobes and rat dura mater during insertion. Journal of Materials Science: Materials in Medicine, vol. 26, no 2, pp. 1-9.

27. Srinivasan, Akhil, et al. (2015). Microchannel-based regenerative scaffold for chronic peripheral nerve interfacing in amputees. Biomaterials, vol. 41, pp. 151-165.

28. Gore, Russell K., et al. (2015). Functional recordings from awake, behaving rodents through a microchannel based regenerative neural interface. Journal of neural engineering, vol. 12, no 1, pp. 016017.

29. Fekete, Zoltan. (2015). Recent advances in silicon-based neural microelectrodes and microsystems: a 
review. Sensors and Actuators B: Chemical, vol. 215, pp. 300-315.

30. Torfs, Tom, et al. (2012). Two-dimensional multi-channel neural probes with electronic depth control. IEEE Transactions of Biomedical Circuits and Systems, vol. 5, no 5, pp. 403-412.

31. Prasad, Abhishek; Sanchez, Justin C. (2012). Quantifying long-term microelectrode array functionality using chronic in vivo impedance testing. Journal of neural engineering, vol. 9, no 2, pp. 26-28.

32. Kretzberg, Jutta, Thomas Coors, and Julia Furche. (2009). Comparision of Valley Seeking and TDistributed EM algorithm for Spike Sorting. BMC Neuroscience,Vol 10, No. 1, pp.1. 2009.

33. Shoham, Shy. (2002). Robust clustering by deterministic agglomeration EM of mixture of multivariante T-Distributions. Pattern Recognition, Vol 35, No. 5, pp. 1127-1142.

\section{Authors}

Oralia Nolasco Jáuregui was born in Apulco, Nochistlán, Zacatecas, México. She received the B.E. degree in Electronics from Universidad Autónoma de Aguascalientes in 2010. She studied the specialization in Electrical Engineering from CINVESTAVGuadalajara. She is currently working for the D. Sc.

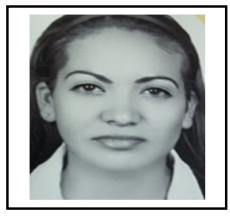

José L. Leyva Montiel was born in Toloyucan, Estado de México. Several of his designs He received awards internationals of his researches and He is SNI level 2 (National Research System), he received the award of Premio Nacional de Ciencias y Artes of the area of Technology and Design by the Secretaría de Educación Pública (SEP) in 2009.

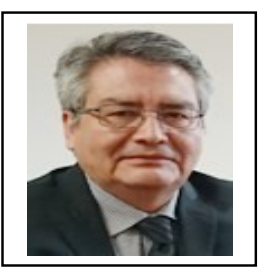

